\title{
Expectativas y valoración del pensamiento crítico de estudiantes de ingeniería de la Universidad Continental, Huancayo
}

\author{
Expectations and assessment of the critical thinking of engineering \\ students of the Universidad Continental, Huancayo
}

\author{
Pepe Raúl De la Cruz Sullca ${ }^{1^{*}}$ \\ ${ }^{1}$ Universidad Continental
}

\section{RESUMEN}

El objetivo de este trabajo fue determinar el nivel de relación que tienen las expectativas y la valoración del pensamiento crítico de los estudiantes de ingeniería de la Universidad Continental. Para cumplir con este propósito se ha seleccionado una muestra de 168 estudiantes de ingeniería de la Universidad Continental; quienes participaron en una encuesta, haciendo uso de la Escala Motivacional de Pensamiento Crítico. Los resultados muestran respecto a las expectativas del pensamiento crítico según edad, que los pertenecientes al grupo etario de 18 a 23 años tienen una alta expectativa sobre el desarrollo de su pensamiento crítico; y sólo 7 de los encuestados manifiestas tener una baja expectativa; asimismo respecto a la valoración del pensamiento crítico según edad, tienen una alta valoración sobre el desarrollo de su pensamiento crítico un total de 165 estudiantes; y una baja valoración sólo 3 de los estudiantes; los estudiantes que tienen una baja valoración tienen la edad de 18, 20 y 28 años respectivamente; analizada por sexo, especialidad y semestre no se encuentran diferencias significativas. En conclusión, se tiene que el nivel de relación de las expectativas de los estudiantes con respecto al desarrollo de su pensamiento crítico y la valoración del pensamiento crítico que poseen es alta, positiva y significativa; esto se infiere a partir de la chicuadrado calculado que es 70 , este valor es alta y tiene una correlación positiva; asimismo su p-valor es 0 por tanto es significativa.

Palabras clave: Pensamiento crítico, expectativas, valoración.

\begin{abstract}
The objective of this work was to determine the level of relationship between the expectations ans the assessment of critical thinking of engineering students of the Universidad Continental. To fulfill this porpuse, a sample of 168 engineering students of Universidad Continental has been selected; who participed in a survey, making use of the Motivational Critical Thinking scale. Thre results show respect to the expectations of critical thinking according to age, that those belonging to the age group of 18 to 23 years have a high expectation about the development of their crititcal thinking; and only 7 ofthe respondent only The results show respect to the expectations of critical thinking according to age, that those belonging to the age group of 18 to 23 years have a high expectation about the development of their critical thinking; and only 7 of the respondents stated that they had a low expectation; also regarding the assessment of critical thinking according to age, a total of 165 students have a high rating on the development of their critical thinking; and a low rating only 3 of the students; Students who have a low rating are 18, 20 and 28 years old respectively; analyzed by sex, specialty and semester no significant differences are found. In conclusion, the level of relationship of students' expectations regarding the development of their critical thinking and the assessment of critical thinking they have is high, positive and significant; this is inferred from the calculated chi-square which is 70 , this value is high and has a positive correlation; likewise its $p$-value is 0 therefore it is significant.
\end{abstract}

Keywords: Critical thinking, expectations, assessment.

Historial del artículo:

Recibido, 11 de junio 2017; aceptado, 21 de junio de 2017; disponible en línea, 25 de junio de 2017

* Magister en Educación, con mención en Investigación y Docencia Superior en la Universidad Nacional Mayor de San Marcos. Docente de la Universidad Continental.

Correo: rdelacruzs@continental.edu.pe 


\section{INTRODUCCIÓN}

El desarrollo del pensamiento crítico es una de las tareas sustantivas de la educación superior. La investigación del pensamiento crítico es fundamental para cumplir dicha tarea. En este contexto, la presente investigación de ocupa de las expectativas y valoraciones del pensamiento crítico que tienen los estudiantes de ingeniería de la Universidad Continental. El problema del presente estudio es: ¿¿ué nivel de relación tienen las expectativas y la valoración del pensamiento crítico de los estudiantes de ingeniería de la Universidad Continental? A partir de esta interrogante se formula el siguiente objetivo: determinar el nivel de relación que tienen las expectativas y la valoración del pensamiento crítico de los estudiantes de ingeniería de la Universidad Continental. La hipótesis de investigación es el siguiente: el nivel de relación que tienen las expectativas y la valoración del pensamiento crítico de los estudiantes de ingeniería de la Universidad Continental es alta, positiva y significativa.

Los estudios sobre el pensamiento crítico son abundantes. De ellos hemos seleccionado algunos que nos sirva de base para el presente estudio. La literatura nos proporciona una amplia gama de definiciones y una variedad de puntos de vista sobre el pensamiento crítico. De acuerdo con el diccionario de la RAE, el pensamiento crítico es el análisis objetivo y la evaluación de un problema para formar un juicio, mientras que de acuerdo con Merchán (2012), "el pensamiento crítico se trata de tener habilidades de cierto tipo". Es interesante notar que la primera definición sugiere el pensamiento crítico como sustantivo, mientras que el último punto de vista describe el pensamiento crítico como un conjunto de habilidades. Muchas de las definiciones señalan al pensamiento crítico como un proceso: "actividad cognitiva que está asociada con el uso de la mente" (Olivares y Heredia, 2012, p. 19); "pensamiento analítico y argumental" (Charles, 2014); "asunciones de caza" (Paul y Elder, 2005); "una forma de autodesarrollo" (Moreno-Pinado, 2015), "un acto disciplinado" (Morales, 2014).

Lipman (1988) define al pensamiento crítico como un pensamiento hábil y responsable que conduce al buen juicio porque se basa en criterios, es autocorrectivo y es sensible al contexto (Milla, 2012). López et al (2014) ha definido el pensamiento crítico como, "pensamiento razonable y reflexivo que se centra en decidir qué creer o hacer".

En este contexto, los autores citados precisan que es importante que el pensamiento crítico no se confunda con otras formas de pensamiento. Por supuesto, el pensamiento crítico y el razonamiento tienen una posición privilegiada con respecto al conocimiento sobre otros procesos como la resolución de problemas, el pensamiento creativo y la toma de decisiones. Por eso, el pensamiento crítico es parte de la familia de formas estrechamente relacionadas de pensamiento de orden superior constituidas como sistema que contiene a la resolución de problemas, la toma de decisiones y el pensamiento creativo.

Así, pensar en formas analíticas, críticas y evaluativas significa usar procesos mentales como la atención, la categorización, la selección y el juicio. El análisis, la implicación, la síntesis, la evaluación, la aplicación y el uso de habilidades para planificar el resultado en una situación se consideran fundamentales para el pensamiento crítico. Los estudiantes pueden aprender a reconocer y usar las habilidades apropiadamente y hacer que su pensamiento sea más efectivo.

El pensamiento crítico también tiene un valor positivo, por ejemplo, la superación personal; autocorrectivo; autodesarrollo o el pensamiento crítico como beneficioso tanto para uno mismo como para todos los demás. En resumen, existen varias definiciones de pensamiento crítico en la literatura. Es significativo que ninguno de los teóricos esté en desacuerdo con la complejidad del pensamiento crítico y su relación con un número ilimitado de comportamientos en un número ilimitado de situaciones.

Existen dos razones para conservar o adoptar varias definiciones sobre el pensamiento crítico, en lugar de trabajar únicamente con una definición: 1) mantener una idea de las diversas dimensiones del pensamiento crítico que resaltan las definiciones alternativas, y 2) ayudarse a escapar de las limitaciones de cada uno. En conjunto, la literatura revisada sugiere que el pensamiento crítico es una lista de habilidades cognitivas, que guían a las personas a tomar decisiones y juicios informados para todas las situaciones de la vida.

El pensamiento crítico es una habilidad cognitiva que requiere de un marco teórico o conceptual para dominar y monitorear los diferentes escenarios de la vida teórica y práctica de los hombres (9). Además, al igual que cualquier otra habilidad, el pensamiento crítico puede ser poseído por un individuo en mayor o menor grado (Facione, 2007). Esto es la razón por el que se impone una gran responsabilidad al alumno para conocer y dominar el pensamiento crítico (Puente, et al, 2007).

Porque el pensamiento crítico es un proceso autodirigido, autodisciplinado, de autocontrol y autocorrectivo, se convierte en una capacidad esencial para desarrollar una actividad, proceso 0 procedimiento. Por eso, el pensamiento crítico se convierte en una habilidad transferible al currículo y al contexto de la vida real y es imperativo que el estudiante tenga la inclinación por estas habilidades. Las disposiciones de los estudiantes pueden influir en 
la forma en que usan las habilidades de pensamiento crítico. No es suficiente enseñar habilidades o que los estudiantes desarrollen habilidades, si simplemente no están dispuestos a usarlos. Un pensador crítico exitoso es aquel que puede pensar, actuar y crecer de acuerdo con sus fundadas y buenas razones y es quién puede articular y hacer explícitos esos motivos y razones.

Ser experto en el pensamiento crítico implica conocer, tal vez implícitamente o sin la capacidad de articular este conocimiento, tanto un conjunto de procedimientos como cuándo aplicar esos procedimientos. Las personas que han desarrollado estas disposiciones tienen muchas más probabilidades de aplicar apropiadamente sus habilidades de pensamiento crítico tanto en su vida personal como en su vida diaria que aquellos que han dominado las habilidades pero no están dispuestas a usarlas.

La "motivación" personal es el principal facilitador del pensamiento crítico, el "disfrute" del pensamiento crítico es el principal factor que lleva al individuo al pensamiento crítico. A veces la necesidad de resolver un problema complejo o de hacer un juicio crucial en la vida o un 'dilema' conduce al pensamiento crítico. Y esta es la razón fundamental para evaluar la escala motivacional del pensamiento crítico de los estudiantes de ingeniería de la Universidad Continental.

\section{MATERIAL Y MÉTODOS}

La investigación es de tipo básica o cognitiva. Y del nivel relacional. Su diseño es descriptivo-correlacional. El método es el método científico.

La población de estudio fueron los estudiantes de ingeniería de la Universidad Continental de la sede Huancayo que hacen un total de 2450 estudiantes. La muestra es de 168 estudiantes que representan las distintas especialidades y semestres académicos.

En el estudio se relacionan las variables expectativas y valoraciones del pensamiento crítico. La técnica de recolección de datos es encuesta. El instrumento de recolección de datos es la escala motivacional del pensamiento crítico (Valenzuela, 2007).

La escala "Escala motivacional de pensamiento crítico - empc" según Valenzuela (2007) consiste en:

"Expresa tu grado de acuerdo con las siguientes afirmaciones, según el siguiente criterio: Totalmente en

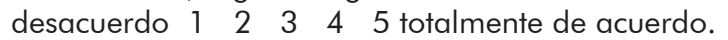

\section{Expectativa}

- Para mi es importante ser bueno(a) para resolver problemas.
- Cuando se trata de razonar correctamente, soy mejor que la mayoría de mis compañeros.

- Me siento capaz de comprender todo lo relacionado con pensar de manera rigurosa.

- Soy capaz de aprender a pensar de manera rigurosa.

- Soy capaz de aprender a razonar correctamente mejor que la mayoría de mis compañeros.

\section{Valoración (Importancia)}

- Para mi es importante aprender a razonar correctamente.

- Para mi es importante ser bueno para razonar.

- Para mi es importante utilizar correctamente mis habilidades intelectuales.

- Para mi es importante ser bueno para resolver problemas.

\section{Utilidad}

- Pensar de manera crítica me servirá para ser un buen profesional.

- Pensar de manera crítica será útil para mi futuro.

- Pensar de manera rigurosa es útil para la vida cotidiana.

- Pensar de manera crítica es útil para otras asignaturas o cursos.

\section{Interés}

- Me gusta razonar bien, antes de decidir algo.

- Me gusta aprender cosas que mejoran la calidad de mi pensamiento.

- Me gusta pensar críticamente.

- Me gusta razonar de manera rigurosa.

\section{Costo}

- Si tengo un problema que requiere razonar de manera crítica estoy dispuesto a sacrificar tiempo de otras actividades.

- Estoy dispuesto a sacrificar bastante tiempo y esfuerzo a sacrificar por mejorar mi manera de razonar.

- Vale la pena invertir tiempo y esfuerzo para tener un pensamiento crítico".

El procesamiento de análisis de datos se realiza en función de las variables y para la prueba de hipótesis se ha calculado la prueba chi-cuadrado.

\section{RESULTADOS}

Los resultados de la aplicación de la escala motivacional del pensamiento crítico se muestran en las siguientes tablas.

En la tabla 1 tenemos las expectativas del pensamiento 
crítico según sexo. Tienen una alta expectativa sobre el desarrollo de su pensamiento crítico en un total de 125 pertenecientes al sexo masculino y a 36 del sexo femenino. Y tiene una baja expectativa sólo 7 varones y ninguna mujer tiene dicha expectativa.

Tabla 1. Expectativas del pensamiento crítico según sexo.

\begin{tabular}{lccc}
\hline \multirow{2}{*}{ Sexo } & \multicolumn{2}{c}{$\begin{array}{c}\text { Expectativas del } \\
\text { pensamiento crítico }\end{array}$} & Total \\
\cline { 2 - 3 } & Baja & Alta & \\
\hline Masculino & 7 & 125 & 132 \\
Femenino & 0 & 36 & 36 \\
\hline Total & 7 & 161 & 168 \\
\hline Fuente: Encuesta sobre el pensamiento crítico a los estudiantes de la \\
\multicolumn{3}{l}{ Universidad Continental, 2017. }
\end{tabular}

En la tabla 2 tenemos las expectativas del pensamiento crítico según edad. Tienen una alta expectativa sobre el desarrollo de su pensamiento crítico los pertenecientes al grupo etario de 18 a 23 años. Y sólo 7 de los encuestados manifiestas tener una baja expectativa.

Tabla 2. Expectativas del pensamiento crítico según edad.

\begin{tabular}{cccc}
\hline \multirow{2}{*}{ Edad } & \multicolumn{2}{c}{$\begin{array}{c}\text { Expectativas del } \\
\text { pensamiento crítico }\end{array}$} & Total \\
\cline { 2 - 3 } & Baja & Alta & \\
\hline 17 & 1 & 4 & 5 \\
18 & 1 & 23 & 24 \\
19 & 0 & 24 & 24 \\
20 & 1 & 30 & 31 \\
21 & 0 & 15 & 15 \\
22 & 1 & 18 & 19 \\
23 & 1 & 17 & 18 \\
24 & 0 & 9 & 9 \\
25 & 0 & 6 & 6 \\
26 & 1 & 0 & 1 \\
27 & 0 & 3 & 3 \\
28 & 1 & 3 & 4 \\
29 & 0 & 3 & 3 \\
30 & 0 & 3 & 3 \\
34 & 0 & 1 & 1 \\
35 & 0 & 2 & 2 \\
\hline Total & 7 & 161 & 168 \\
\hline
\end{tabular}

Fuente: Encuesta sobre el pensamiento crítico a los estudiantes de la Universidad Continental, 2017.

En la tabla 3 tenemos las expectativas del pensamiento crítico según especialidad. Tienen una alta expectativa sobre el desarrollo de su pensamiento crítico un total de 161 estudiantes. Y tiene una baja expectativa sólo 7 de los estudiantes.

En la tabla 4 tenemos las expectativas del pensamiento crítico según semestre académico. Tienen una alta
Tabla 3. Expectativas del pensamiento crítico según especialidad.

\begin{tabular}{lcrr}
\hline \multirow{2}{*}{ Especialidad } & \multicolumn{2}{c}{$\begin{array}{c}\text { Expectativas del } \\
\text { pensamiento crítico }\end{array}$} & Total \\
\cline { 2 - 3 } & Baja & Alta & \\
\hline Ing. Sistemas e Informática & 1 & 19 & 20 \\
Ing. Civil & 6 & 107 & 113 \\
Ing. Empresarial & 0 & 12 & 12 \\
Ing. de Minas & 0 & 2 & 2 \\
Ing. Mecatrónica & 0 & 20 & 20 \\
Ing. Ambiental & 0 & 1 & 1 \\
\hline Total & 7 & 161 & 168
\end{tabular}

Fuente: Encuesta sobre el pensamiento crítico a los estudiantes de la Universidad Continental, 2017.

expectativa sobre el desarrollo de su pensamiento crítico un total de 161 estudiantes. Y tiene una baja expectativa sólo 7 de los estudiantes.

Tabla 4. Expectativas del pensamiento crítico según semestre.

\begin{tabular}{cccc}
\hline \multirow{2}{*}{ Semestre } & \multicolumn{2}{c}{$\begin{array}{c}\text { Expectativas del } \\
\text { pensamiento crítico }\end{array}$} & Total \\
\cline { 2 - 3 } & Baja & Alta & \\
\hline I & 0 & 3 & 3 \\
II & 1 & 6 & 7 \\
III & 0 & 10 & 10 \\
IV & 1 & 70 & 71 \\
V & 1 & 24 & 25 \\
VI & 3 & 35 & 38 \\
VII & 0 & 9 & 9 \\
VIII & 1 & 4 & 5 \\
\hline Total & 7 & 161 & 168
\end{tabular}

Fuente: Encuesta sobre el pensamiento crítico a los estudiantes de la Universidad Continental, 2017.

En la tabla 5 tenemos la valoración del pensamiento crítico según sexo. Tienen una alta valoración sobre el desarrollo de su pensamiento crítico un total de 165 estudiantes. Y tiene una baja valoración sólo 3 de los estudiantes.

Tabla 5. Valoración del pensamiento crítico según sexo.

\begin{tabular}{lccc}
\hline \multirow{2}{*}{ Sexo } & \multicolumn{2}{c}{$\begin{array}{c}\text { Valoración del } \\
\text { pensamiento crítico }\end{array}$} & Total \\
\cline { 2 - 3 } & Baja & Alta & \\
\hline Masculino & 3 & 129 & 132 \\
Femenino & 0 & 36 & 36 \\
\hline Total & 3 & 165 & 168 \\
\hline
\end{tabular}

Fuente: Encuesta sobre el pensamiento crítico a los estudiantes de la Universidad Continental, 2017.

En la tabla 6 tenemos la valoración del pensamiento crítico según edad. Tienen una alta valoración sobre 
Tabla 6. Valoración del pensamiento crítico según edad.

\begin{tabular}{cccc}
\hline \multirow{2}{*}{ Edad } & \multicolumn{2}{c}{$\begin{array}{c}\text { Valoración del } \\
\text { pensamiento crítico }\end{array}$} & Total \\
\cline { 2 - 3 } & Baja & Alta & \\
\hline 17 & 0 & 5 & 5 \\
18 & 1 & 23 & 24 \\
19 & 0 & 24 & 24 \\
20 & 1 & 30 & 31 \\
21 & 0 & 15 & 15 \\
22 & 0 & 19 & 19 \\
23 & 0 & 18 & 18 \\
24 & 0 & 9 & 9 \\
25 & 0 & 6 & 6 \\
26 & 0 & 1 & 1 \\
27 & 0 & 3 & 3 \\
28 & 1 & 3 & 4 \\
29 & 0 & 3 & 3 \\
30 & 0 & 3 & 3 \\
34 & 0 & 1 & 1 \\
35 & 0 & 2 & 2 \\
\hline Total & 3 & 165 & 168 \\
\hline
\end{tabular}

Fuente: Encuesta sobre el pensamiento crítico a los estudiantes de la Universidad Continental, 2017.

el desarrollo de su pensamiento crítico un total de 165 estudiantes. Y tiene una baja valoración sólo 3 de los estudiantes. Debe anotarse que los estudiantes que tienen una baja valoración tienen la edad de 18, 20 y 28 años respectivamente.

En la tabla 7 tenemos la valoración del pensamiento crítico según especialidad. Tienen una alta valoración sobre el desarrollo de su pensamiento crítico un total de 165 estudiantes. Y tiene una baja valoración sólo 3 de los estudiantes. Debe anotarse que 2 estudiantes de Ingeniería Civil manifiestan su baja valoración.

En la tabla 8 tenemos la valoración del pensamiento crítico según semestre. Tienen una alta valoración sobre el desarrollo de su pensamiento crítico un total

Tabla 7. Valoración del pensamiento crítico según especialidad.

\begin{tabular}{lcrr}
\hline \multirow{2}{*}{ Especialidad } & \multicolumn{2}{c}{$\begin{array}{c}\text { Valoración del } \\
\text { pensamiento crítico }\end{array}$} & Total \\
\cline { 2 - 3 } & Baja & Alta & \\
\hline Ing. Sistemas e Informática & 1 & 19 & 20 \\
Ing. Civil & 2 & 111 & 113 \\
Ing. Empresarial & 0 & 12 & 12 \\
Ing. de Minas & 0 & 2 & 2 \\
Ing. Mecatrónica & 0 & 20 & 20 \\
Ing. Ambiental & 0 & 1 & 1 \\
\hline Total & 3 & 165 & 168 \\
\hline
\end{tabular}

Fuente: Encuesta sobre el pensamiento crítico a los estudiantes de la Universidad Continental, 2017. de 165 estudiantes. Y tiene una baja valoración sólo 3 de los estudiantes. Debe anotarse que tiene una baja valoración un estudiante del semestre IV y dos estudiantes del VI semestre académico.

Tabla 8. Valoración del pensamiento crítico según semestre.

\begin{tabular}{cccc}
\hline \multirow{2}{*}{ Semestre } & \multicolumn{2}{c}{$\begin{array}{c}\text { Valoración del } \\
\text { pensamiento crítico }\end{array}$} & Total \\
\cline { 2 - 3 } & Baja & Alta & \\
\hline I & 0 & 3 & 3 \\
II & 0 & 7 & 7 \\
III & 0 & 10 & 10 \\
IV & 1 & 70 & 71 \\
V & 0 & 25 & 25 \\
VI & 2 & 36 & 38 \\
VII & 0 & 9 & 9 \\
VIII & 0 & 5 & 5 \\
\hline Total & 3 & 165 & 168 \\
\hline
\end{tabular}

Fuente: Encuesta sobre el pensamiento crítico a los estudiantes de la Universidad Continental, 2017.

La tabla 9 relaciona la variable expectativas y valoración del pensamiento crítico. Aquí tenemos que 161 estudiantes tienen una alta expectativa $y$ valoración del desarrollo de su pensamiento crítico. Hay 4 estudiantes que tienen baja expectativa, pero alta valoración de su pensamiento crítico. Y sólo 3 estudiantes tienen baja expectativa y baja valoración del desarrollo de su pensamiento crítico.

Es evidente que existe fuerte correlación entre dichas variables. Ahora queremos saber que tan significativa es, para ello calculamos la prueba chi-cuadrado.

Tabla 9. Relación entre expectativas y valoración del pensamiento crítico.

\begin{tabular}{lcrc}
\hline \multirow{2}{*}{$\begin{array}{c}\text { Expectativas del } \\
\text { pensamiento crítico }\end{array}$} & \multicolumn{2}{c}{$\begin{array}{c}\text { Valoración del } \\
\text { pensamiento crítico }\end{array}$} & Total \\
\cline { 2 - 3 } & \multicolumn{1}{c}{ Baja } & Alta & \\
\hline Baja & 3 & 4 & 7 \\
Alta & 0 & 161 & 161 \\
\hline Total & 3 & 165 & 168 \\
\hline
\end{tabular}

Fuente: Encuesta sobre el pensamiento crítico a los estudiantes de la Universidad Continental, 2017.

En la tabla 10 la chi-cuadrado calculado es 70, este valor es alta correlación. Y observamos que su p-valor es 0 . Si el p-valor hubiera sido mayor que 0,05 tendríamos que rechazar la hipótesis de investigación. Como este valor ha sido menor aceptamos la hipótesis de investigación en el sentido de que el nivel de relación que tienen las expectativas y la valoración del pensamiento crítico de los estudiantes de ingeniería de la Universidad Continental es alta, positiva y significativa. 
Tabla 10. Prueba Chi cuadrado.

\begin{tabular}{lccccc}
\hline & Valor & gl & $\begin{array}{c}\text { Sig. asintótica } \\
\text { (bilateral) }\end{array}$ & $\begin{array}{c}\text { Sig. exacta } \\
\text { (bilateral) }\end{array}$ & $\begin{array}{c}\text { Sig. exacta } \\
\text { (unilateral) }\end{array}$ \\
\hline Chi-cuadrado de Pearson $^{\text {Corrección por continuidad }}$ b & 70,255 & 1 & 0,000 & & \\
Corrán & 47,943 & 1 & 0,000 & & \\
Razón de verosimilitudes & 20,538 & 1 & 0,000 & & 0,000 \\
$\begin{array}{l}\text { Estadístico exacto de Fisher } \\
\text { Asociación lineal por lineal }\end{array}$ & 69,836 & 1 & 0,000 & & \\
N de casos válidos & 168,000 & & & & \\
\hline
\end{tabular}

\section{DISCUSIÓN}

La educación superior tiene como objetivo preparar a los graduados con habilidades de pensamiento crítico para hacer frente a realidades complejas e inéditas. La educación debe elevar la comprensión humana acerca de quiénes somos y qué podemos hacer. Esto es idéntico a la de "ser crítico" y el "hacer crítico". El pensamiento crítico es una parte inherente de la educación. El desarrollo del pensamiento crítico es fundamentalmente de carácter colaborativo y se basa en la práctica o las intervenciones de la acción en el mundo.

Cuando los estudiantes aprenden a ver las cosas a través de su habilidad de pensamiento crítico, desarrollan una postura crítica orientado al mundo del conocimiento. El pensamiento crítico es una reconstitución del conocimiento, reconstitución de uno mismo y reconstitución del mundo. El conocimiento crítico siempre aspira no solo a ser crítico, sino a ser reflexivo y autorreflexivo. Todas estas características han sido detectadas en los estudiantes de ingeniería de la Universidad Continental.

También es evidente que el pensamiento crítico de cualquier tipo nunca es universal en ningún individuo; todos están sujetos a episodios de pensamiento indisciplinado o irracional. Por lo tanto, el pensamiento crítico suele ser una cuestión de grado y depende, entre otras cosas, de la calidad y la profundidad de la experiencia en un dominio determinado de pensamiento o con respecto a una clase particular de preguntas o cuestiones a las que se enfrenta la persona.

Finalmente, en la investigación se ha encontrado que en los estudiantes, el pensamiento crítico se ve afectado por el andamiaje de los grupos de pares, es decir, edad, sexo, semestre o especialidad. El pensamiento crítico está situado socialmente; es un proceso de aprendizaje social y en función de sus características y peculiaridades de cada uno de los estudiantes. La naturaleza emergente de la cognición grupal o colectiva, en la creación de sentido, ofrece una nueva dirección potencial para la investigación del pensamiento crítico. Pues se ha determinado que la participación de los estudiantes en grupos pequeños ofrece los momentos más interesantes para aprender a pensar críticamente. La diversidad de respuestas y el intercambio animado de ideas entre compañeros en pequeños grupos beneficia a los estudiantes. El trabajo grupal, el trabajo con pares o el trabajo en parejas fomentan el pensamiento crítico en los estudiantes. Todo esto se infiere del análisis de los resultados de la aplicación de la escala motivacional de pensamiento crítico a los estudiantes de ingeniería de la Universidad Continental.

Las conclusiones son:

El nivel de relación que tienen las expectativas y la valoración del pensamiento crítico de los estudiantes de ingeniería de la Universidad Continental es alta, positiva y significativa porque la chi-cuadrado calculado es 70 , este valor es alta y positiva correlación y el $p$-valor es 0 por tanto es significativa.

La evaluación del pensamiento, en general, y del pensamiento crítico, en particular, implica verificar el pensamiento a través de los siguientes estándares intelectuales: claridad, precisión, relevancia, significado, lógica, profundidad, amplitud, precisión e imparcialidad. Los estándares intelectuales deben ser considerados como un "conjunto de filtros" para evaluar el pensamiento. Por tanto, reflexionar y mejorar las habilidades del pensamiento crítico implica juzgar cuándo uno está o no está funcionando bien, o qué tan bien o como sea posible, y también implica considerar las maneras de mejorar el desempeño de uno mismo en cuanto al pensamiento crítico.

\section{REFERENCIAS BIBLIOGRÁFICAS}

Merchán, M. (2012). Cómo desarrollar los procesos del pensamiento crítico mediante la pedagogía de la pregunta. Actualidades Pedagógicas, (59), 119 146.

Olivares, S. \& Heredia, Y. (2012). Desarrollo del pensamien to crítico en ambientes de aprendizaje basado en problemas en estudiantes de educación 
superior. Revista Mexicana de Investigación Educativa, 17(54), 759-778.

Charles, D. (2014). Desarrollo de la competencia del pensamiento crítico utilizando aprendizaje basado en proyectos en nivel profesional. Tesis inédita de maestría, Universidad Tecvirtual, Tecnológico de Monterrey.Toluca, México.

Paul, R. y Elder, L. (2005). La miniguía para el pensamiento crítico conceptos y herramienta. Recuperado de http://www.criticalthinking.org/ resources/

Moreno-Pinado, W. E. (2015). Estrategia didáctica para desarrollar el pensamiento crítico en estudiantes del quinto de secundaria. Recuperado de http:// repositorio.usil.edu.pe/

Morales, L. (2014). El pensamiento crítico en la teoría educativa contemporánea. Actualidad Investigativas en Educación, 14(2), 4-23. doi:10.15517/aie.v14i2.14833

Milla, M. (2012). Pensamiento crítico en estudiantes de quinto de secundaria de los colegios de Carmen de la Legua Callao (Tesis de maestría). Universidad San Ignacio de Loyola, Lima.
López, M., Et al. (2014). Estructura de conocimiento conceptual, memoria de trabajo y comprensión de textos de ciencias: un estudio con alumnos de secundaria REICE. Revista Iberoamericana sobre Calidad, Eficacia y Cambio en Educación, 12(3), 57-72.

Castellanos, D. (2007). Reflexiones metacognitivas y estrategias de aprendizaje. La Habana: Editorial Pueblo y Educación.

Facione, P. (2007). Pensamiento crítico: ¿̇Qué es y por qué es importante? Recuperado de http://www. eduteka.org/pensamientoCríticoFacione.php.

Puente, A., Moya, J. y Mayor, L. (2007). Cognición y aprendizaje. Madrid: Pirámide

Valenzuela, J. (2007). Escala Motivacional de Pensamiento Crítico. Universidad de Salamanca. doi: 10.13140/RG.2.1.2063.3049. 